\title{
Zoning Design for Handwritten Numeral Recognition
}

\author{
G. DIMAURO, S. IMPEDOVO, G. PIRLO, A. SALZO \\ Dipartimento di Informatica - Università di Bari \\ Via Orabona, 4 - 70126 Bari - Italy
}

\begin{abstract}
This paper presents a new approach for zoning design. The approach is based on a techinque which detects the most discriminant image regions by the analysis of feature distributions, and obtains the zoning by an iterative zone-growing process. An application to handwritten numeral recognition is also reported showing the effectiveness of the proposed approach.
\end{abstract}

\section{Introduction}

As it is well known, different writing conditions and personal styles produce enormous variability in handwritten numerals and this also among patterns of the same class [1]. To solve the problem and improve the recognition many papers recently appeared in literature have stressed the importance of using regional information extracted by zoning techniques $[2,3,4,5]$.

When zoning is used, handwritten numerals are first normalized to an image of standard size, called control box, successively, the control box is divided into zones which contains information on specific parts of the numerals. Up to now, the selection of an effective zoning, i.e. the way in which the control box must be divided into smaller regions, is carried out on the basis of a priori experience or intuitive motivations. In the simplest approaches, the control box is divided into zones of equal size: for instance, the paper of Baptista Kulkarni presents a system for handwritten numeral recognition based on geometrical and topological features in which the control box is divided by a $3 \times 3$ grid into 9 zones of equal size [2]. In the paper of B.Hussain and M.R.Kabuka the control box is divided by a $4 \times 4$ grid into 16 zones of equal size [3]. In other approaches, the number and the size of the zones depend on the feature under consideration or also on the characteristics of the pattern to be recognized. For instance, the paper of P.Ahmed and C.Y.Suen presents a recognition system based on end-points, concavities and holes; the position of the end-points is captured by dividing the control box into 16 zones of equal size, the position of concavities and holes is captured by dividing the control box into $32 \times 32$ zones [4]. In the paper of M.Y.Chen, A. Kundu and J.Zhou the control box is nonuniformly divided into three zones along the vertical directions according to specific characteristics of the pattern [5].

This paper presents a new technique for automatic zoning design. Specifically, in Section 2 some basic criteria for zoning design are discussed. Section 3 presents the new technique. Section 4 reports the algorithm for handwritten numeral recognition used for the experimental test. Section 5 shows the experimental results obtained by a traditional zoning approach and with the new technique. The conclusion is reported in Section 6. 


\section{Zoning Design Criteria}

A zoning $Z=\left\{z_{1}, \ldots, z_{j,}, \ldots, z_{M}\right\}$ of a control box $\mathrm{B}$, is a partition of $\mathrm{B}$, i.e. $Z$ is a set of regions $\left\{z_{1}, \ldots, z_{i,}, \ldots, z_{M}\right\}$ of $\mathrm{B}$ for which it results:

$$
\begin{aligned}
& \forall \mathrm{j}=1,2, \ldots, M \quad \mathrm{z}_{\mathrm{j}} \neq \varnothing \\
& \forall \mathrm{j}_{1}, \mathrm{j}_{2}=1,2, \ldots, M, \quad \mathrm{j}_{1} \neq \mathrm{j}_{2}: \mathrm{z}_{\mathrm{j}_{1}} \cap \mathrm{z}_{\mathrm{j}_{2}}=\varnothing \\
& \bigcup_{\mathrm{j}=1}^{\mathrm{M}} \mathrm{z}_{\mathrm{j}}=\mathrm{B}
\end{aligned}
$$

Of course, if no assumption is done on the connectivity of each zone, for a control box of W-pixel in width and H-pixel in height, $\left(\begin{array}{c}W x H \\ M\end{array}\right)$ different zonings can be defined and the selection of the best one for a given problem is very difficult.

However, when zoning is used to extract regional information for recognition, the set $C=\left\{c_{l}, \ldots, c_{k}, \ldots, c_{m}\right\}$ of different classes and the feature set $F=\left\{f_{l}, \ldots, f_{i}, \ldots, f_{n}\right\}$ are generally known. In this case, the zoning design can be regarded as an optimization problem which consists in detecting the zoning $Z^{*}=\max _{Z} S\left(z_{1}, \ldots, z_{1}, \ldots, z_{M}\right)$, being $S\left(z_{1}, \ldots, z_{j}, \ldots, z_{M}\right)$ an optimality function (see Fig. 1).

Of course, several optimality functions can be considered both at feature level, based on discrimination capabilities or information measures, and at decision level, if the classification responses obtained at zone level are integrated by a combination method like Bayes [6], Majority Vote [7], Behaviour-Knowledge Space [8], Dempster-Shafer [9], and so on.

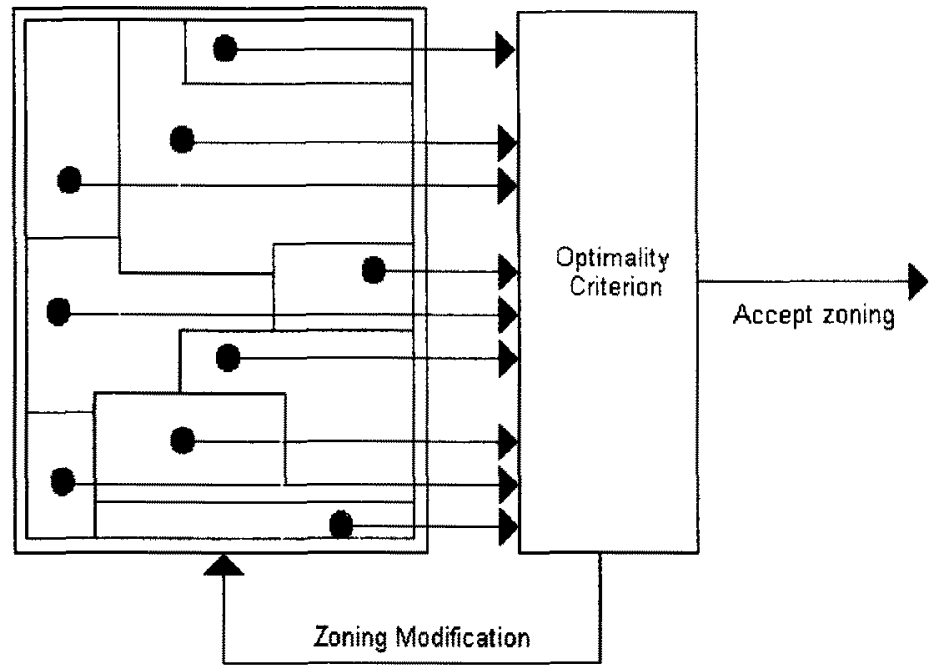

Fig. 1 Zoning design technique 
In this paper the zoning $Z^{*}$ is selected according to the criterion $Z^{*}=$ $\max _{z}\left\{S\left(z_{1}\right)+\ldots+S\left(z_{j}\right)+\ldots+S\left(z_{M}\right)\right\}$ where, for each $\mathrm{j}=1,2, \ldots, \mathrm{M}, S\left(z_{j}\right)$ is a measure of the discrimination capability of the $j$-th zone, and the number of zones $M$ is fixed. For this purpose, a reference set of patterns $X=\left\{x_{1}, \ldots, x_{t}, \ldots, x_{N}\right\}$ is used to estimate the feature distributions for the set of classes $C$. Specifically, for each feature $f_{i}$, the statistical distribution of its position in the control box is estimated by the values:

$$
\mathrm{X}_{\mathrm{C}_{\mathrm{k}}}(\mathrm{s}, \mathrm{r})=\left\{\mathrm{x}_{\mathrm{t}} \in \mathrm{X} \mid \mathrm{x}_{\mathrm{t}} \in \mathrm{C}_{\mathrm{k}} \text {, and } f_{\mathrm{i}} \text { has been detected in } \mathrm{x}_{\mathrm{t}} \text { at pixel }(\mathrm{s}, \mathrm{r})\right\} \text {, }
$$

which are computed for each point $(\mathrm{s}, \mathrm{r})$ of the control box, and

$$
\mathrm{X}_{\mathrm{C}_{\mathrm{k}}}\left(\mathrm{Z}_{\mathrm{j}}\right)=\sum_{\{B(s, r)\} \in z_{j}} X_{C_{k}}(s, r)
$$

which are computed for each zone $Z_{j}$.

Therefore, for $\mathrm{j}=1,2, \ldots, \mathrm{M}, S\left(z_{j}\right)$ is defined as:

$$
S\left(z_{j}\right)=\sqrt{\frac{1}{m} \sum_{k=1}^{m}\left[p_{k}\left(z_{j}\right)-\mu\left(z_{j}\right)\right]^{2}}
$$

where:

$$
\mu\left(z_{j}\right)=\frac{1}{m} \sum_{k=1}^{m} p_{k}\left(z_{j}\right)
$$

and $\forall \mathrm{k}=1,2, \ldots, \mathrm{m}$ :

$$
p_{k}=\frac{\operatorname{card}\left(X_{C_{k}}\left(z_{j}\right)\right)}{\sum_{h=1}^{m} \operatorname{card}\left(X_{C_{h}}\left(z_{j}\right)\right)} \quad \text { if } \sum_{h=1}^{m} \operatorname{card}\left(X_{C_{h}}\left(z_{j}\right)\right)>0
$$

otherwise $p_{k}=0$.

\section{The Zoning Design Technique}

The zoning desing technique here proposed follows an iterative zone-growing process which consists of three fundamental steps: local analysis, starting point detection and zone growing. 


\subsection{Local Analysis}

The first step is devoted to the evaluation of discriminant characteristics of features at pixel level. For this purpose, let be $f_{i}$ the feature under consideration, for each pixel of coordinates $(\mathrm{s}, \mathrm{r})$ of the control box, the value

$$
S(s, r)=\sqrt{\frac{1}{m} \sum_{k=1}^{m}\left[p_{k}(s, r)-\mu(s, r)\right]^{2}}
$$

is computed, where, $\forall \mathbf{k}=1,2, \ldots, \mathbf{m}$ :

and

$$
\mu(s, r)=\frac{1}{m} \sum_{k=1}^{m} p_{k}(s, r) .
$$

$p_{k}(s, r)=\frac{\operatorname{card}\left(X_{C_{k}}(s, r)\right)}{\sum_{h=1}^{m} \operatorname{card}\left(X_{C_{h}}(s, r)\right)}$

$$
\text { if } \sum_{h=1}^{m} \operatorname{card}\left(X_{C_{h}}(s, r)\right)>0
$$

otherwise $p_{k}(s, r)=0$.

\subsection{Starting point detection}

In the second step the starting point of the first zone $z_{1}$ is detected. For this purpose the point with maximum discrimination capability is selected, i.e. the point $B\left(\mathrm{~s}^{*}, \mathrm{r}^{*}\right)$ for which

$$
S\left(s^{*}, r^{*}\right)=\max _{\{(s, r)\} \in B} S(s, r)
$$

\subsection{Zone growing}

The third step is devoted to the iterative process of zone growing. Let $z_{p}$ be a zone, an expanded version of $z_{p}$ is given by including in $z_{p}$ one of its adjacent pixels. Among the expanded versions of $z_{p}$, the zone $z_{p}$ with the greatest standard deviation value $S\left(z_{p}\right)$ is selected. At each iteration, let be $\left\{z_{1}, z_{2}, \ldots, z_{p}, \ldots, z_{j}\right\}$ the set of zones already defined, the zone expansion occurs by considering the new set of zones $\left\{z_{1}, z_{2}, \ldots, z_{p}, \ldots, z_{j}\right\}$ for which it results:

$$
S\left(z_{p}\right)-S\left(z_{p}\right)=\max _{v=1,2, \ldots, j}\left\{S\left(z_{v}\right)-S\left(z_{v}\right)\right\} .
$$

Of course, if the number of zones is less than $M$ (i.e. $j<M$ ), then a new zone $\mathrm{z}_{j+1}=\left\{\mathrm{B}\left(\mathrm{s}^{\prime}, \mathrm{r}^{\prime}\right)\right\}$ can be created in place of growing an existing zone. This case occurs when $B\left(s^{\prime}, r^{\prime}\right)$ is a local maximum not included into an existing zone, and it results:

$$
S\left(z_{j+1}\right)>S\left(z_{p}\right)-S\left(z_{p}\right)
$$


Table 1 shows a simple example of the procedure of zoning design. Two classes are considered: "A" (28 patterns) and "B" (36 patterns). The distribution of the feature for the two classes, in a small control box of $5 \times 5$ pixel size, is reported in Table $1 \mathrm{a}$ and $1 \mathrm{~b}$ respectively. Table 1.1 reports the result of the local analysis step. The result of the starting point detection step is reported in Table 1.2. In Table $1.5 \mathrm{a}$ new zone is created. The final configuration of the two zones is shown in Table 1.10.

\begin{tabular}{|l|l|l|l|l|}
\hline 0 & 0 & 6 & 2 & 0 \\
\hline 0 & 0 & 4 & 3 & 0 \\
\hline 0 & 4 & 3 & 3 & 0 \\
\hline 0 & 0 & 0 & 3 & 0 \\
\hline 0 & 0 & 0 & 0 & 0 \\
\hline \multicolumn{6}{|c|}{ Table 1a }
\end{tabular}

\begin{tabular}{|c|c|c|c|c|}
\hline 0 & 0 & 3 & 1 & 0 \\
\hline 0 & .5 & 1 & 1.5 & 0 \\
\hline 0 & 2 & 0 & .5 & 0 \\
\hline 0 & 0 & 0 & 1.5 & 3 \\
\hline 0 & 0 & 0 & 3 & 3.5 \\
\hline
\end{tabular}

Table 1.1

\begin{tabular}{|c|c|c|c|c|}
\hline 0 & 0 & 3 & 1 & 0 \\
\hline 0 & .5 & 1 & 1.5 & 0 \\
\hline 0 & 2 & 0 & .5 & 0 \\
\hline 0 & 0 & 0 & 1.5 & 3 \\
\hline 0 & 0 & 0 & 3 & \\
\hline
\end{tabular}

Table 1.2

\begin{tabular}{|l|l|l|l|l|}
\hline 0 & 0 & 3 & 1 & 0 \\
\hline 0 & .5 & 1 & 1.5 & 0 \\
\hline 0 & 2 & 0 & .5 & 0 \\
\hline 0 & 0 & 0 & 1.5 & 3 \\
\hline 0 & 0 & 0 & \multicolumn{2}{|c}{} \\
\hline
\end{tabular}

Table 1.3

\begin{tabular}{|l|l|l|c|c|}
\hline 0 & 0 & 3 & 1 & 0 \\
\hline 0 & .5 & 1 & 1.5 & 0 \\
\hline 0 & 2 & 0 & .5 & 0 \\
\hline 0 & 0 & 0 & 1.5 & \\
\hline 0 & 0 & 0 & & \\
\hline
\end{tabular}

Table 1.4

\begin{tabular}{|c|c|c|c|c|}
\hline 0 & 0 & IIIIII & 1 & 0 \\
\hline 0 & .5 & 1 & 1.5 & 0 \\
\hline 0 & 2 & 0 & .5 & 0 \\
\hline 0 & 0 & 0 & 1.5 & \\
\hline 0 & 0 & 0 & & \\
\end{tabular}

Table 1.5

\begin{tabular}{|l|l|l||c|c|}
\hline 0 & 0 & & 1 & 0 \\
\hline 0 & .5 & & \\
\hline 0 & 2 & 0 & .5 & 0 \\
\hline 0 & 0 & 0 & & \\
\hline 0 & 0 & 0 & \\
\hline
\end{tabular}

Table 1.6

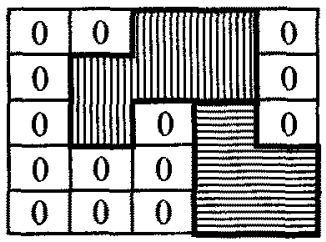

Table 1.10

\begin{tabular}{|c|c|c|c|c|}
\hline 0 & 0 & 0 & 0 & 0 \\
\hline 0 & 1 & 2 & 0 & 0 \\
\hline 0 & 0 & 3 & 4 & 0 \\
\hline 0 & 0 & 0 & 6 & 6 \\
\hline 0 & 0 & 0 & 6 & 7 \\
\hline \multicolumn{5}{|c|}{} \\
\hline
\end{tabular}

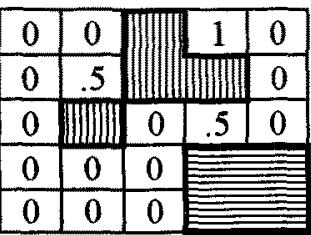

Table 1.7

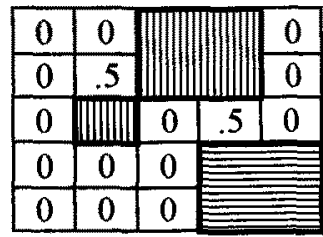

Table 1.8

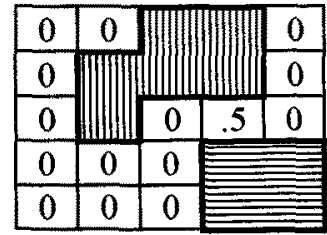

Table 1.9 


\section{The Recognition Algorithm}

The zoning technique has been applied to a system for handwritten digit recognition. The system is based on a syntactic approach [4]. Three kinds of structural features are detected (see Table 2): cavities, end-points and holes. A dedicated holograph is used to store the statistical distribution of the position of each feature in the training patterns [10]. For this purpose a zoning is used to easily obtain information on the position of the features. During the recognition phase, let be $x_{t}$ a test pattern described by the set of features $\left\{\left(f_{q}, z_{q}\right)\right\}_{q=1,2, \ldots,}$, where $\left(f_{q}, z_{q}\right)$ means that the feature $f_{q}$ has been detected in the zone $z_{q}$. In this case for each $\mathrm{k}=1,2, \ldots, \mathrm{m}$, the values

$$
\mathbf{R}_{C_{k}}=\frac{1}{Q} \sum_{q=1}^{Q} P_{C_{k}}\left(f_{q}, z_{q}\right)
$$

are computed, where $P_{C k}$ is the probability that the feature $f_{q}$ is in $z_{q}$, for a pattern of the class $C_{k}$. The test pattern $x_{t}$ is then classified as belonging to the class $C_{k}$ for which $R_{C k}$ assumes the greatest value.

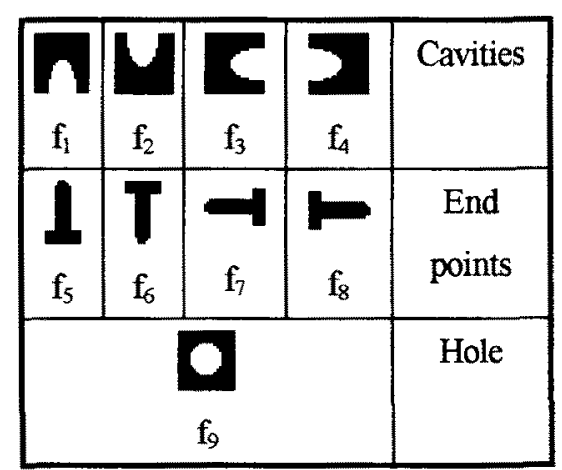

Table 2 The Feature Set

\section{Experimental Results}

The experimental test has been carried out, in the field of handwritten numeral recognition using a training set of 18468 patterns and a test set of 2213 patterns (directories BR and BS of the CEDAR database).

Two different zoning have been considered: (a) the first one obtained by the traditional partition of the control box into 9 zones of equal size (see Fig. 2a), (b) the second zoning obtained by the technique proposed in this paper using a number of zones $\mathrm{M}=9$ (see Fig. $2 b$ ). 
Table 3 reports the experimental results for the two zonings which show the efficacy of the technique proposed for zoning design. In fact, on the entire test database, the recognition rate achieved by the new zoning (b) is more than $20 \%$ better than the recognition rate obtained by the traditional zoning (a).

\begin{tabular}{|l|l|l|}
\hline G & H & I \\
\hline D & E & F \\
\hline A & B & C \\
\hline
\end{tabular}

(a)

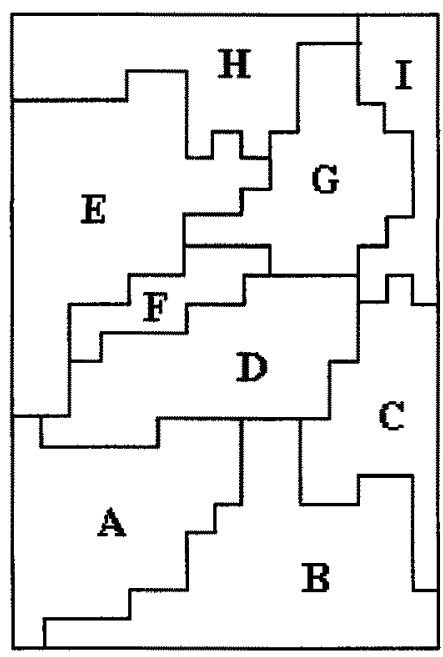

(b)

Fig. 2 (a) Traditional zoning, (b) New zoning for $f_{\text {g. }}$

\begin{tabular}{|c||c||c||}
\hline Class & $\begin{array}{c}\text { Recognition Rate } \\
\text { Zoning "a" }\end{array}$ & $\begin{array}{c}\text { Recognition Rate } \\
\text { Zoning "b" }\end{array}$ \\
\hline 0 & $62.6 \%$ & $73.5 \%$ \\
\hline 1 & $80.2 \%$ & $86.0 \%$ \\
\hline 2 & $84.0 \%$ & $83.6 \%$ \\
\hline 3 & $81.5 \%$ & $94.0 \%$ \\
\hline 4 & $76.5 \%$ & $88.0 \%$ \\
\hline 5 & $56.0 \%$ & $83.0 \%$ \\
\hline 6 & $64.2 \%$ & $86.4 \%$ \\
\hline 7 & $63.0 \%$ & $79.1 \%$ \\
\hline 8 & $59.7 \%$ & $77.1 \%$ \\
\hline 9 & $60.8 \%$ & $76.6 \%$ \\
\hline Tot. & $68.5 \%$ & $82.5 \%$ \\
\hline
\end{tabular}

Table 3 Experimental Results 


\section{Conclusion}

It this paper a new technique for the automatic design of zoning is presented. Statistical distributions of features are used to design zones with high discriminant capabilities. The experimental results, carried out in the field of handwritten numeral recognition, show the effectiveness of the new approach.

\section{References}

1. C.Y.Suen, M. Berthod, S. Mori, "Computer Recognition of Handprinted Characters: The State of the Art", Proc. of IEEE, 68 (4), pp. 469-483, 1980.

2. G.Baptista, K.M.Kulkarni, "A high accuracy algorithm for recognition of handwritten numerals", Pattern Recognition 4, pp.287-291, 1988.

3. B. Hussain and M. R. Kabuka, "A Novel Feature Recognition Neural Network and its Application to Character Recognition", IEEE Transactions on Pattern Analysis Machine Intelligence, vol. 16, no. 1, pp. 98-106, Jan 1994.

4. P. Ahmed and C. Y. Suen, "Computer Recognition of Totally Uncostrained Handwritten Zip Codes", International Journal of Pattern Recognition and Artificial intelligence, vol. 1, no. 1, pp. 1-15, 1987.

5. M. Yen Cen, A. Kundu and J. Zhou, "Off-Line Handwritten Word Recognition Using a Hidden Markov Model Type Stochastic Network ", IEEE Transactions on Pattern Analysis Machine Intelligence, vol. 16, no. 5, pp. 481-496, May 1994.

6. Ley $\mathrm{Xu}$, Adam Krzyzak, Ching Y-Suen, "Methods of Combining Multiple Classifiers and Their Applications to Handwriting Recognition", IEEE Transaction on Systems, Man and Cybernetics, Vol. 22, N. 3, 1992, pp. 418-435.

7. Hull, T.K.Ho, J. Favata, V. Govindaraju, S. Srihari, "Combination of Segmentation-based and Wholistic Handwritten Word Recognition Algorithms", in From Pixels to Feature III - Frontiers in Handwriting Recognition, S. Impedovo and J.C. Simon eds., Elsevier Publ., pp. 261-272, 1992.

8. Huang, C.Y. Suen, "An Optimal Method of Combining Multiple Classifiers for Unconstrained Handwritten Numeral Recognition", Proc. of IWFHR-3, Buffalo, NY, 1993, pp. 11-20.

9. Y. Lu, F. Yamaoka, "Integration of Handwritten Digit Recognition results using Evidential Reasoning", Proc. of IWFHR-4, 1994, pp. 456-463.

10. G. Dimauro, S. Impedovo, G. Pirlo, A. Salzo, "A multi-expert system to handwritten digit recognition", in Progress in Handwriting Recognition, S. Impedovo Ed., World Scientific, 1997, pp. 363-367. 